\title{
A Proposed Method for Optimizing the Spectral Discernibility of Engineered Point- spread Functions for Localization Microscopy
}

\author{
Jason T. Martineau ${ }^{1}$, Rajesh Menon ${ }^{2}$, Erik M. Jorgensen ${ }^{3}$ and Jordan Gerton ${ }^{1 *}$ \\ 1. University of Utah, Department of Physics and Astronomy, Salt Lake City, USA. \\ 2. University of Utah, Department of Electrical and Computer Engineering, Salt Lake City, USA. \\ 3. University of Utah, Department of Biology, Salt Lake City, USA. \\ * Corresponding author: jgerton@physics.utah.edu
}

Localization microscopy has become an established tool for investigating protein distributions within cells at resolutions around $20 \mathrm{~nm}$ [1-3]. An outstanding challenge within the field stems from spectral width of the fluorescent markers used to tag proteins of interest - generally about $50 \mathrm{~nm}$ FWHM. This width decreases the number of protein species it is possible to tag and simultaneously observe without incurring unacceptable cross-talk between spectral channels. Given how dynamic and interconnected all of the functions of a live cell are, this is a significant limitation for future live-cell imaging applications. Additionally, markers with spectral centeres in the range of 650-750 $\mathrm{nm}$ produce better signal to noise ratios than do bluer dyes due to the low interaction between red light and the cellular environment. Thus, multi-color experiments with red dyes would be ideal in this sense but their spectral widths and the resulting cross-talk currently make such experiments impossible.

Recently, we and others [4-7] have begun using point-spread function (PSF) engineering, rather than traditional filters, to separate spectral channels. By introducing a phase mask into the Fourier plane (FP) of a microscope (Fig. 1.c), the PSF can be made very sensitive to emission spectra. In this way we hope to image many fluorescent tags simultaneously with negligible cross-talk. To optimize such a PSF, we introduce a figure of merit (FOM) called pixel confusion, which characterizes the probability that a given pixel will have the same photon count for two different emission spectra.

More specifically, suppose two PSFs, $X_{1}$ and $X_{2}$, are centered on the same region of a camera. If $X_{1}, X_{2}$ have different spectra then their PSFs will differ based on the design of the phase plate. If the photon count, $x_{i}$, of the $i^{t h}$ pixel between the measurements of $X_{1}$ and $X_{2}$ is the same, then we say that the $i^{t h}$ pixel is confused. Assuming Poissonian detection statistics, the average probability of confusion (POC), over all of the pixels $K$ of the region of interest, is:

$$
\left\langle P\left(X_{1}=X_{2}\right)\right\rangle=\frac{1}{K} \sum_{i=1}^{K} \sum_{x_{i}=0}^{\infty} \operatorname{poiss}\left(\mu_{1, i}, x_{i}\right) \operatorname{poiss}\left(\mu_{2, i}, x_{i}\right) \rightarrow \frac{1}{K} \sum_{i=1}^{K} \exp \left(-\mu_{1, i}-\mu_{2, i}\right) I_{0}\left(2 \sqrt{\mu_{1, i} \mu_{2, i}}\right)
$$

Above, $\mu_{1, i}, \mu_{2, i}$ are the expected photon rates for $X_{1}, X_{2}$ at the $i^{\text {th }}$ pixel. The function $I_{0}()$ is a modified Bessel function of the first kind, zero order and poiss $\left(\mu_{l, i}, x_{i}\right)$ denotes the Poisson distribution with rate, $\mu_{l, i}$ and argument, $x_{i}$. We propose that minimizing the POC between $X_{1}, X_{2}$ will yield a phase mask that allows us to distinguish between their respective specra. To test this, we optimized a phase mask for the spectra of four commonly used organic dyes, simultaneously. This involved averaging the POC over all unique pairs $(l, m)=(m, l)$ for $\left\langle P\left(X_{l}=X_{m}\right)\right\rangle, \ni m, l \in[1,4]$. These spectra - Alexa Fluors 647, 660, 680, 700 [8] - have a high degree of overlap (Fig. 1.h). After minimizing the POC, the PSF showed a large improvement in correct spectral identification (Fig. 1.a) over an Airy spot (Fig. 1.b). In the future we will apply the above work to multi-color experiments using red dyes. 
References:

[1] E Betzig et al., Science 313 (2006), p. 1642.

[2] ST Hess, TPK Girirajan and MD Mason, Biophysical Journal 91 (2006), p. 4258.

[3] WE Moerner, Journal of Microscopy 246 (2012), p. 213.

[4] JT Martineau et al., Microscopy and Microanalysis 24 (2018), p. 1364.

[5] Y Shechtman et al., Nature Photonics 10 (2016), p. 590.

[6] LEW Eran Hershko, T Michaeli and Y Shechtman, arXiv:180701637, (2018).

[7] J Broeken, B Rieger and S Stallinga, Opt Lett 39 (2014), p. 3352.

[8] ThermoFisher Spectraviewer, https://www.thermofisher.com/us/en/home/life-science/cellanalysis/labeling-chemistry/fluorescence-spectraviewer.html (accessed February 17, 2019).

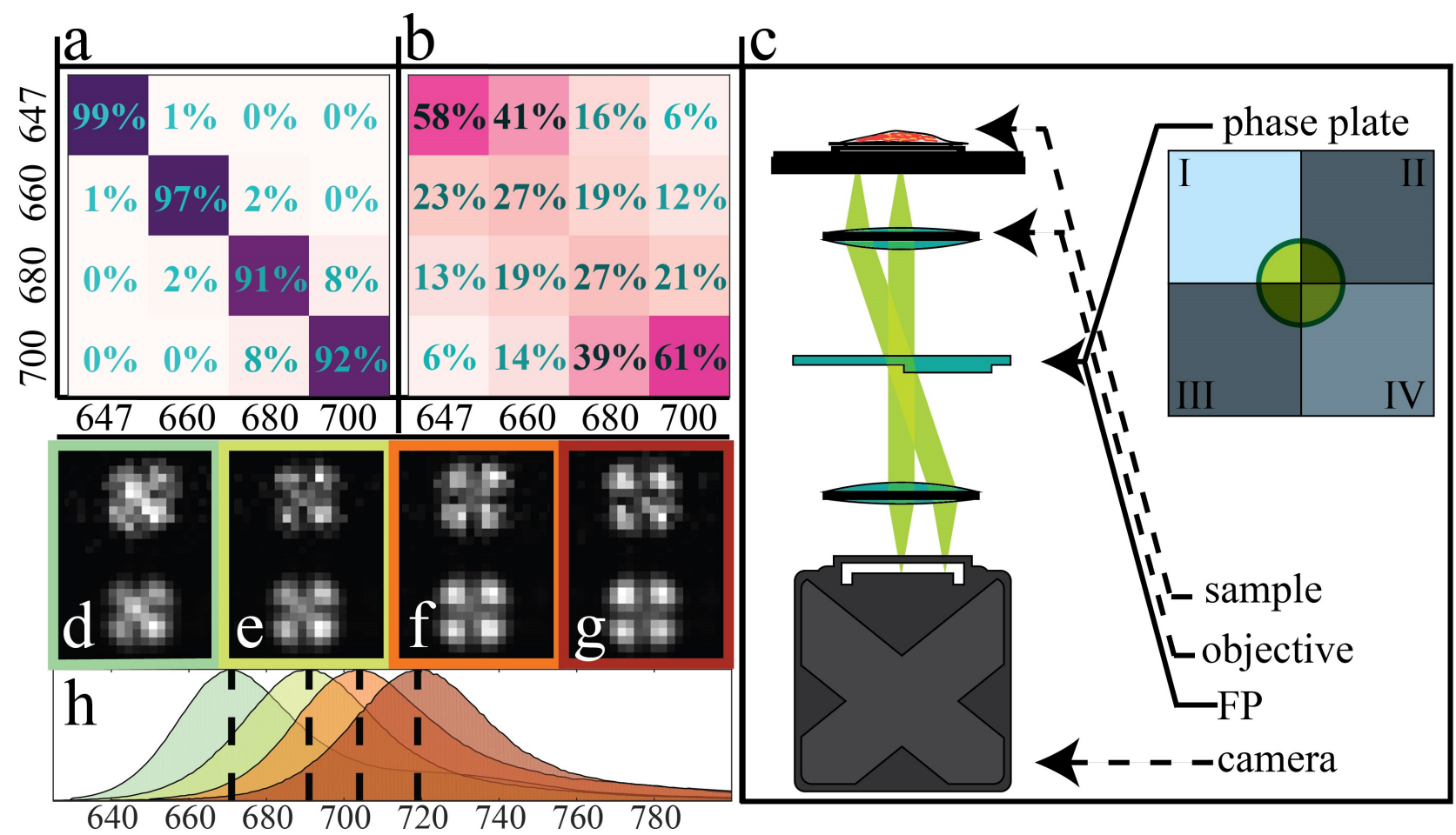

Figure 1. Panel (a): truth table (TT) for results of Monte Carlo spectral identification. Each PSF was simulated using a weighted sum of monochromatic PSFs calculated using a modified Born-Wolf model PSF. This sum was calculated using fluorophore spectra sampled every $10 \mathrm{~nm}$ (17 samples each).The PSFs were simulated with 1,000 photons. The horizontal axis denotes the true simulated spectrum for 1,000 trials and the vertical axis denotes the identified spectrum using a maximum likelihood fitting scheme. Correct identifications reside on the diagonal. For the optimal phase-mask, the rate of correct detection is above 90\% in all cases. Panel (b): the TT for an Airy spot. Correct detection is 61\%, at best. Panel (c): a simple diagram of the detection path of a wide-field microscope with a phase mask. The phase mask design we used in this analysis consists of four regions, I-IV, with an index of refraction of 1.46. For the optimized PSF the thickness of the four regions, I-IV, are as follows: $0 \mu \mathrm{m}, 2.44 \mu \mathrm{m}, 2.44$ $\mu \mathrm{m}, 2.15 \mu \mathrm{m}$. Panels (d-g): simulated average optimal PSFs (bottom) and noisy optimal PSFs (top). The panels are arranged in order of increasing peak wavelength. This minimization was done using particle swarm optimization. Panel (h): the fluorophore spectra used in our simulations. Neighbouring spectral peaks are separated by between 10-20 $\mathrm{nm}$. 